\title{
Influence of Warm Oxide Layer on Wettability and Contact Angle for Heat Transport Devices
}

\author{
A. Takemura ${ }^{1}$, K. Yuki ${ }^{2}$ and A. Sadayuki ${ }^{3}$ \\ 1. Department of Integrated Science and Technology, National Institute of Technology, Tsuyama College, 624-1, Okayama 708-8509, \\ Japan \\ 2. Department of Mechanical Engineering, Faculty of Engineering, Tokyo University of Science, Yamaguchi 756-0084, Japan \\ 3. Department of Mechanical Engineering, National Institute of Technology, Tsuyama College, 624-1, Okayama 708-8509, Japan
}

\begin{abstract}
Recently, high heat density has become a problem in electronic devices. Therefore, high heat-transfer efficiency is required in copper heat exchangers. Improvement of wettability is reported to improve the heat-transfer efficiency. In previous studies, copper oxide layer improves the wettability. In this study, we focus on a copper oxide layer produced under warm conditions (from 200 to $300{ }^{\circ} \mathrm{C}$ ), which are suitable oxidation conditions for improving wettability. Experimental results showed that the surface of the specimens was covered by the oxidation layer and took on a black color. Furthermore, the wettability was improved by the warm copper oxide layer. While, the surface roughness was approximately constant to each warm oxidized specimen. Whereat, the warm oxide layer was observed by SEM (sanning electron microscope). The results from SEM observations showed that the warm copper oxide layer consisted of stacks and combinations of nanoscopic warm oxidation particles. Thus, the warm oxidation layer has nanoscopic surface asperities. It is seemed that these nanoscopic asperities improved the wettability.
\end{abstract}

Key words: Warm oxide layer, heat-transfer efficiency, wettability, contact angle.

\section{Introduction}

Recently, the thermal problems associated with electronic devices have become serious with the increasing demand for downsizing heat exchangers. Therefore, high heat-exchange efficiency is required in heat exchangers. For this reason, high thermal conductivity copper is used extensively in heat exchangers. Further, the improvement in boiling heat-exchange efficiency produced by nanoparticle-assembled bi-porous layers has been proven by recent research $[1,2]$. The nanoparticle-assembled bi-porous layer is produced on the copper heat exchanger surface by evaporation physical vapor deposition. When the nanoparticle-assembled bi-porous layer is produced, the copper heat exchanger is covered by the oxidation layer. In previous studies, it has been reported that the

Corresponding author: Akihiro Takemura, Dr.-Eng., lecturer, research field: metallography.
$\mathrm{Cu}$ oxidation layer reduces wettability. This indicates that, the oxidized heat exchanger has reduced thermal conductivity. However, in previous studies, authors found that the warm copper oxide layer is hydrophilic [3]. If the heat exchanger surface is hydrophilic, the wettability is improved. The warm copper oxide layer has high hydrophilicity, which leads to high evaporation efficiency. Furthermore, the warm copper oxide layer is expected to combine with the nanoparticle-assembled bi-porous layer. It is important to evaluate the association between the copper oxidized layer and wettability.

In previous studies, a $\mathrm{Cu}$ alloy was oxidized above $500{ }^{\circ} \mathrm{C}$. In this study, we focus on the copper oxide layer produced under warm conditions (from 200 to $300^{\circ} \mathrm{C}$ ), which are suitable oxidation conditions for improving wettability. We evaluate the improvement in wettability of a copper oxide layer and its optimum oxidation conditions by estimating the change in contact angle between a water droplet and the copper oxide layer. 


\section{Experimental Methodology}

The material used in this study is oxygen-free copper $(99.99 \mathrm{Cu}$ in $\mathrm{wt} \%)$. The oxygen-free copper specimen plates are square plates with $30 \mathrm{~mm}$ sides and $3 \mathrm{~mm}$ thickness. Furthermore, the surface roughness of the specimens is adjusted to about 0.23 $\mathrm{mm}$ in arithmetic average roughness $\mathrm{Ra}$.

These specimens are oxidized in an electric furnace. Fig. 1 shows the thermal history of the warm oxidation process. First, the electric furnace is heated to the warm oxidation temperature, and then the specimen is put in the electric furnace. The warm oxidation temperatures in this study are 200, 250 and $300{ }^{\circ} \mathrm{C}$. Furthermore, the warm oxidation times are 2, 5, 10, 15, 20, 25, 60 and 180 min. After the specimens are oxidized for the scheduled time, they are ejected from the electric furnace and air-cooled.

After warm oxidation, a droplet of distilled pure water is dropped onto the specimens. The wettability was evaluated by measuring the contact angles of the water droplets on the warm oxidized specimens.

The appearance of the warm oxidized specimens is observed through a stereomicroscope. Furthermore, warm oxidation layers are observed using optical microscopy and SEM. The roughness of the warm oxidized specimens is also measured.

\section{Results and Discussion}

\subsection{Appearance of the Copper Specimen Surface}

Fig. 2 shows the appearance of the bare specimen. The specimen surfaces had polishing tool marks in one direction. These tool marks were created by polishing the surface to make the surface roughness uniform. The surface of the specimen took on a metallic luster. Fig. 3 shows the appearance of the oxidized specimens. The horizontal axis represents the warm oxidation time. The vertical axis represents the warm oxidation temperature. The surface color of the specimens changed as the oxidation time and temperature increased. As oxidation proceeded, the surface of the specimens was covered by the oxidation layer and took on a black color. Changes in the copper specimen surface color are related to the thickness of the oxidation layer $[3,4]$. It is thought that the thickness of the oxidation layer increased owing to warm oxidation.

\subsection{Contact Angles and Improvement of Wettability}

Fig. 4a shows the appearance of the water droplet on the bare copper surface. The bare copper surface was hydrophobic. Fig. 4b shows the appearance of the water droplet on the oxidation layer generated at $300{ }^{\circ} \mathrm{C}$ after $25 \mathrm{~min}$ of oxidation. The warm oxidized surface was hydrophilic. The warm oxidation layers reduced the contact angle. This result shows that the wettability between the water droplet and the oxidation layer improved owing to the warm oxidation layer.

$300{ }^{\circ} \mathrm{C}$

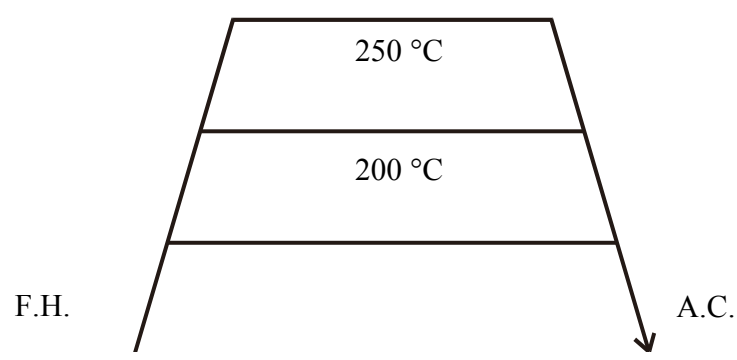

Fig. 1 Thermal history of the warm oxidation process.

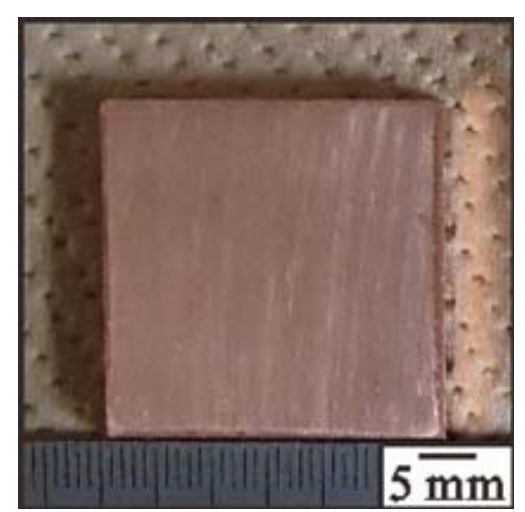

Fig. 2 Appearance of the bare specimen. 


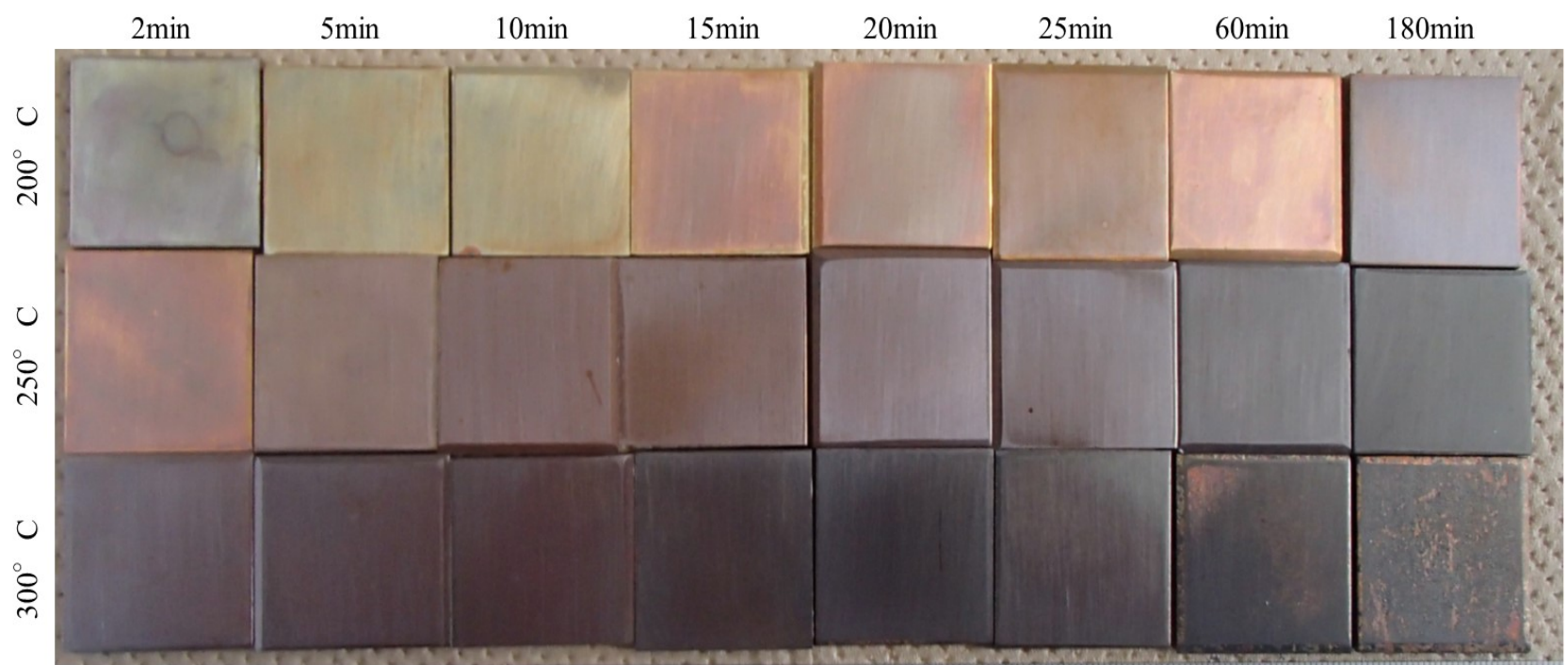

Fig. 3 Appearance of the oxidized specimens.

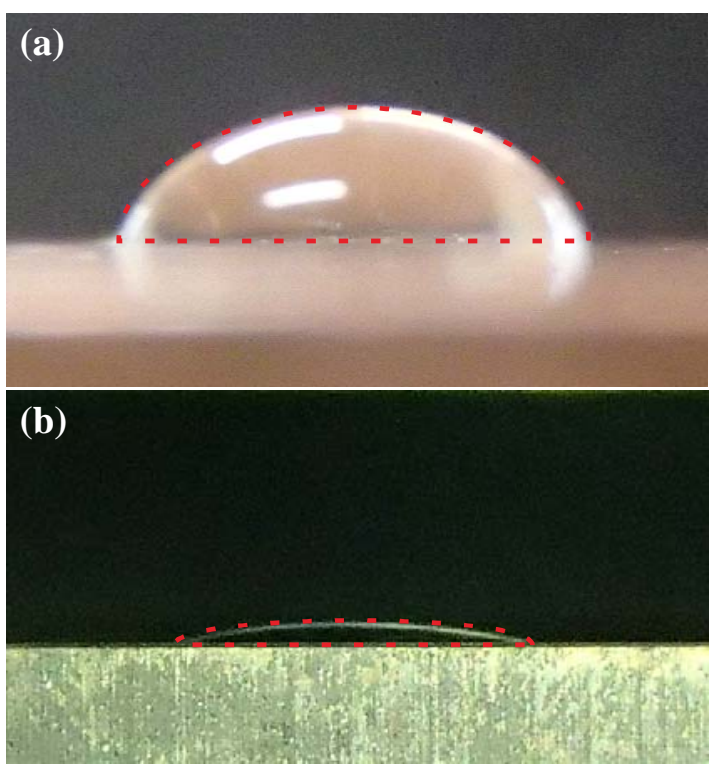

Fig. 4 Appearance of the water droplets: (a) bare surface, (b) 25 min oxidation at $300{ }^{\circ} \mathrm{C}$.

Further, the contact angles were measured from the appearance of the water droplet. Fig. 5 shows the results of the contact angles of the water droplet on the warm oxidized copper specimens. The horizontal axis represents the oxidation time. The vertical axis represents the contact angle. The contact angle of the water droplet on the bare copper surface was $65^{\circ}$. For the oxidation temperatures of 200,250 , and $300^{\circ} \mathrm{C}$, the minimum contact angles were $10.34^{\circ}, 9.06^{\circ}$, and $6.27^{\circ}$, respectively. The contact angles drastically reduced below $10 \mathrm{~min}$ of oxidation. After $10 \mathrm{~min}$ of oxidation, the contact angles remained approximately constant. The warm oxidation time required to obtain high wettability decreased as the warm oxidation temperatures increased. This indicates that, warm oxidation layers were generated by the warm oxidation process, and improved the surface wettability of the copper surface.

\subsection{Roughness of the Copper Specimen Surface}

When the material exhibits high hydrophilicity, wettability improves with increasing roughness. This phenomenon was discovered by Young and Wenzel [5].

$$
r \cos \theta_{r}=\cos \theta=\frac{\gamma_{S}-\gamma_{S L}}{\gamma_{L}}
$$

where, $r$ is the dimensionless roughness factor, which is defined as the ratio of the actual solid surface area to the apparent projection area of a rough surface. $\theta$ is the contact angle between droplet and solid surface, $\theta_{\mathrm{r}}$ is the contact angle between the droplet and rugged solid surface, $\gamma_{S}$ is the surface free energy of the solid, $\gamma_{\mathrm{L}}$ is the surface free energy of the liquid, and $\gamma_{S L}$ is the interfacial free energy between the solid and liquid. 


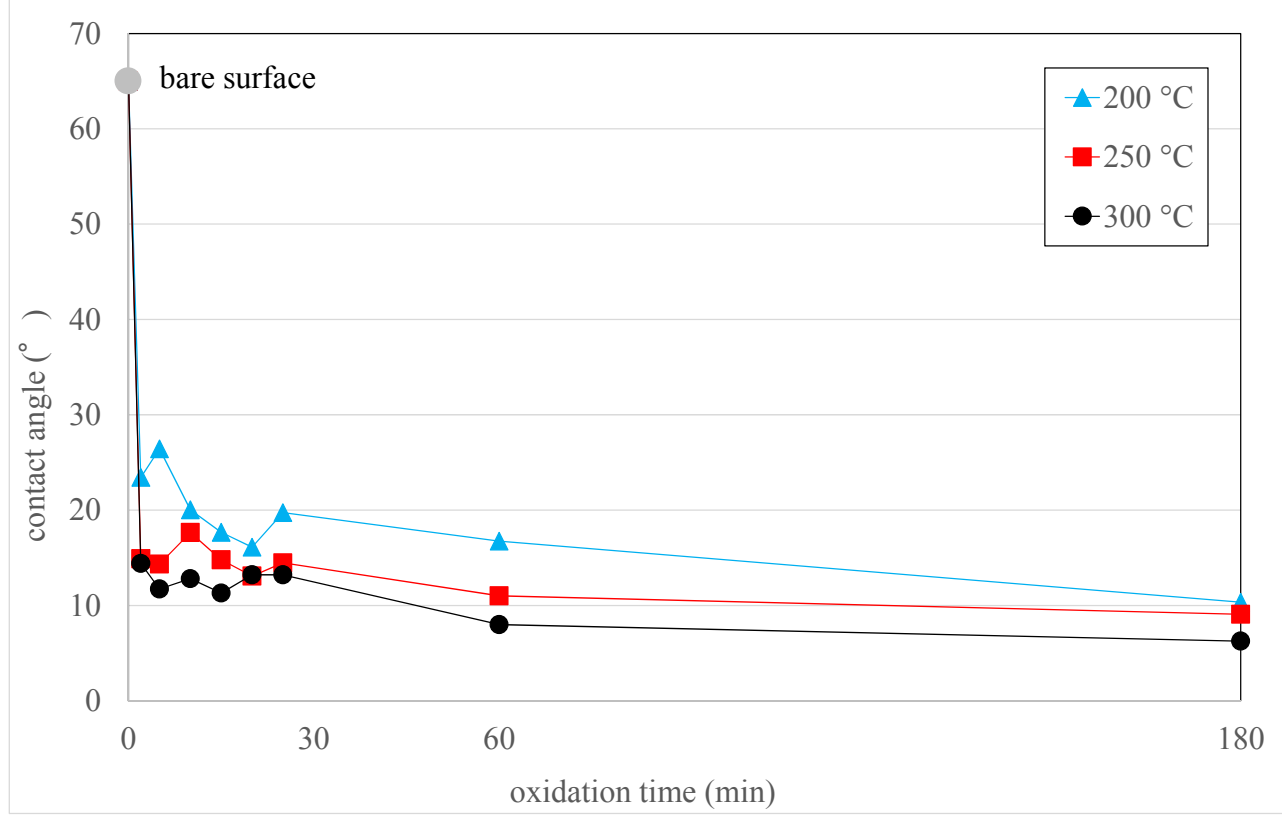

Fig. 5 Results showing the oxidized contact angles of the water droplets on the warm oxidized copper specimens.

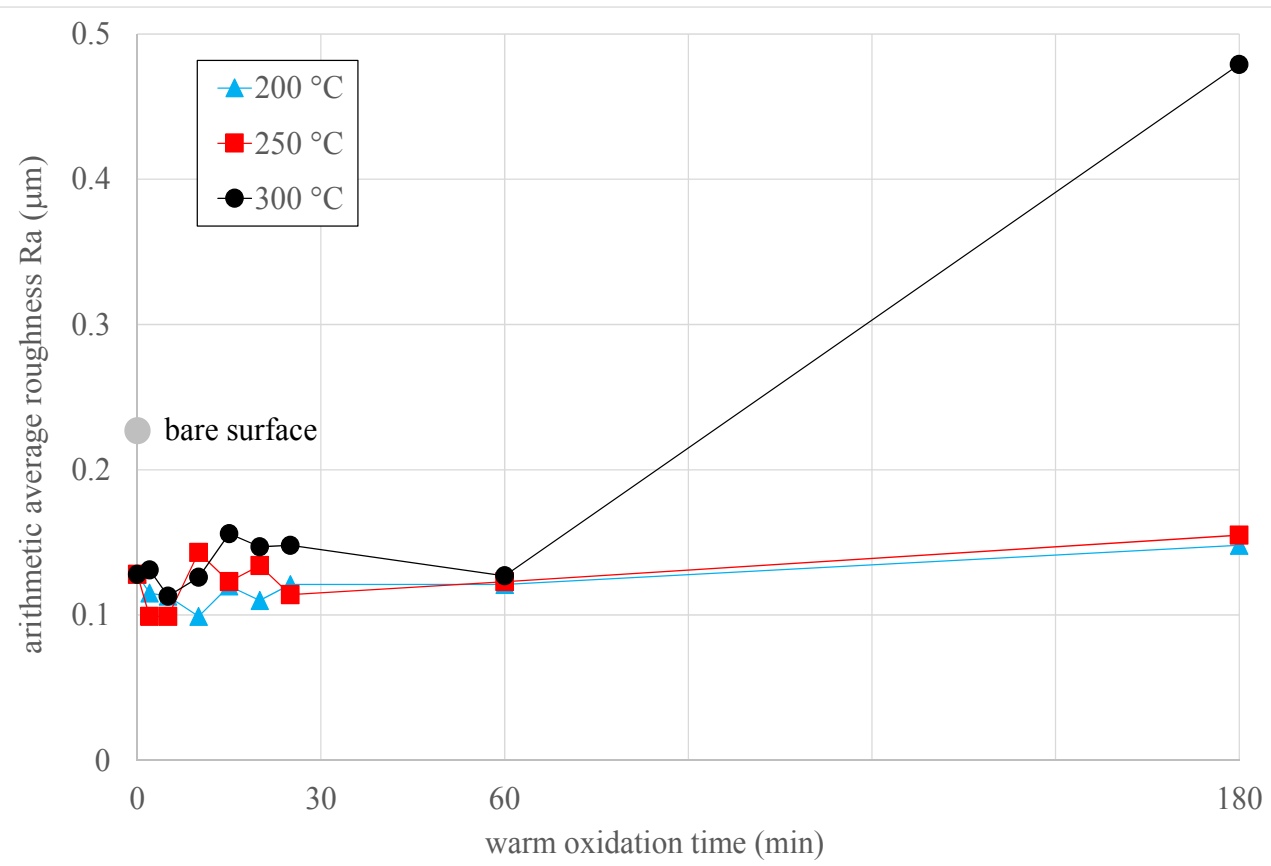

Fig. 6 Results of the surface roughness test.

In the case of $\theta_{\mathrm{r}}$ (from Eq. (1)) $<90^{\circ}$, it is thought that the wettability improved because of the roughness of the copper surface [6]. Therefore, the roughness of the warm oxidized copper specimens is measured. The roughness was measured using the stylus method. Fig. 6 shows the results of the surface roughness test. The horizontal axis represents the oxidation time. The vertical axis represents the arithmetic average roughness $\mathrm{Ra}$. The surface roughness of the bare surface was $0.227 \mu \mathrm{m}$. Even when the warm oxidation time or temperature increased, the surface roughness remained approximately constant. When the warm oxidation time was $180 \mathrm{~min}$ and the warm oxidation temperature was $300{ }^{\circ} \mathrm{C}$, the surface 

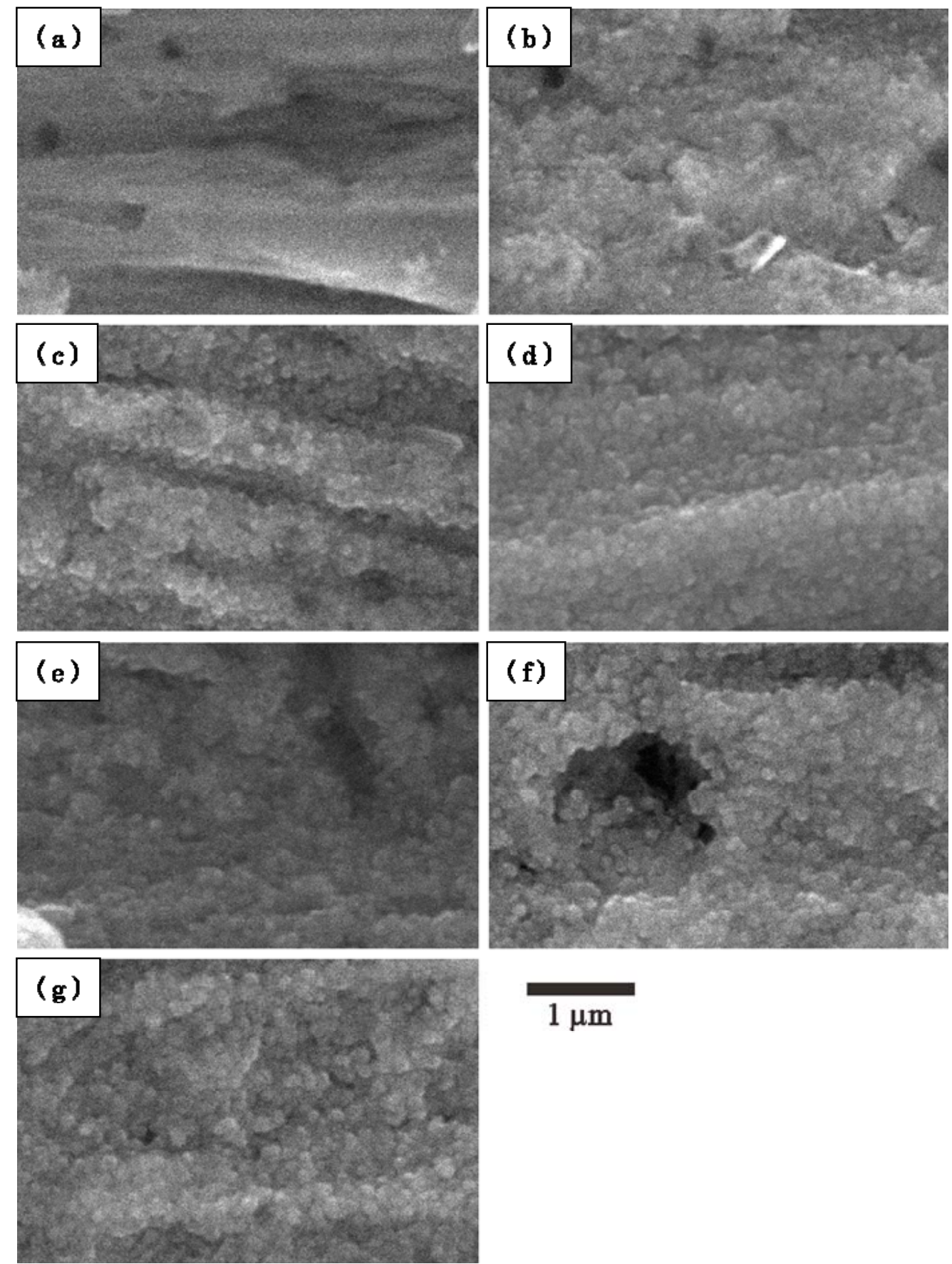

Fig. 7 SEM observation results of specimens oxidized at $200{ }^{\circ} \mathrm{C}$ : (a) bare surface, (b) 30 min oxidized, (c) 60 min oxidized, (d) 90 min oxidized, (e) 120 min oxidized, (f) 150 min oxidized, (g) 180 min oxidized.

roughness increased. This result is attributed to the fact that the surface of the specimens was covered by large $\mathrm{Cu}$-oxides.

\subsection{Microscopic Variations in the Warm Oxidation Layer}

The warm oxidation layer was observed microscopically. Fig. 7 shows the SEM observation results of specimens oxidized at $200{ }^{\circ} \mathrm{C}$. The bare surface had tool marks from machining carried out to adjust the roughness. In the initial-stage of warm oxidation, the fine oxidized particles were observed on the surface of the copper specimens. As oxidation 
progressed, the fine oxidation particles increased and grew larger. These oxidation particles stacked upon one another and eventually formed the oxidation layer $[7,8]$.

Oxidation particles coated the surface of the copper specimens. This process of formation of the oxidation layer seems to be the cause of the surface color change and tarnishing of luster, as shown in Fig. 3 [4].

\subsection{Mechanisms Causing Decrease in Surface Roughness and Improvement of Wettability}

Fig. 2 shows that the tool marks were microscopic. Further, Fig. 7 shows that the oxidation particles were nanoscopic. The nanoscopic oxidation particles filled the microscopic tool marks in. It is thought that roughness was not affected by the filling-in of nanoscopic oxidation particles. Therefore, the wettability of warm oxidized copper specimens was improved. These results indicated that the mechanism for improving wettability is the structure of the warm oxidation layer, and not the roughness of the copper specimen surface. The warm oxidation layer is formed by stacking and combining warm oxidation particles. Thus, the warm oxidation layer has nanoscopic surface asperities. It is thought that the wettability of the warm oxidized copper was improved by the nanoscopic surface asperities. Furthermore, slight porosity was also observed. It is assumed that the local porous structure of the oxidation layer induces capillary action and improves the wettability [9].

\subsection{Formation of the Hydrophilic Oxidation Layer}

As oxidation progresses, the copper oxide layer possibly changes from $\mathrm{CuO}$ to $\mathrm{Cu}_{2} \mathrm{O}$ [4]. Further, there is a possibility that a hydrophilic compound such as $\mathrm{Cu}(\mathrm{OH})_{2}$ is formed, from which hydrated $\mathrm{CuO}$ is formed on the copper surface. It is thought that the $\gamma_{\mathrm{SL}}$ (from Eq. (1)) is reduced by the formation of a hydrophilic compound such as $\mathrm{Cu}(\mathrm{OH})_{2}$. It is important to study the composition and structure of the surface oxidation layer. As the next step, the composition and structure of the surface oxidation layer should be evaluated using SEM-EDX and XRD.

\section{Conclusions}

In this study, we focused on improving the wettability of copper heat exchangers by using a copper oxide layer and evaluated the effects of the oxidation time and temperature on its characteristics. The surface color of the specimens changed as the oxidation time and temperature increased. Furthermore, the contact angles drastically reduced below $10 \mathrm{~min}$ of oxidation. The surface roughness reduced with increasing warm oxidation time and temperature. It was found that the warm oxidation layer was formed by stacking and combining nanoscopic oxidation particles. Nanoscopic oxidation particles filled the surface tool marks in. In addition, it is thought that the warm oxidation layer contained the hydrophilic compound $\mathrm{Cu}(\mathrm{OH})_{2}$.

\section{References}

[1] Yuki, K., Sugiura, T., and Suzuki, K. 2015. "Boiling/Evaporation Characteristics of a Droplet on a Nanoparticles-Assembled Bi-Porous Layer." Transactions of the Japan Society of Mechanical Engineers (in Japanese), 81 (824).

[2] Semenic, T., and Catton, I. 2009. "Experimental Study of Biporous Wicks for High Heat Flux Applications." International Journal of Heat and Mass Transfer 52 (21): 5113-21.

[3] Yuki, K., Fukushima, K., Takemura, A., and Suzuki, K. 2015. "Wettability and Evaporation Enhancement for Heat Transport Devices by High Performance Oxide Layer." In Proceedings of the International Conference on Electronics Packaging (CD-ROM), France.

[4] Goro, I. "Corrosion Science and Corrosion Control Technology." Corona.

[5] de Gennes, P. G., Brochard-Wyart, F., and Quere, D. 2004. Capillarity and Wetting Phenomena: Drops, Bubbles, Pearls, Waves. New York: Springer.

[6] Chi, Y. L., Chun, T. H., and Wang, K. I. 2014. "Effect of Change in Surface Condition Induced by Oxidation on Transient Pool Boiling Heat Transfer of Vertical Stainless Steel and Copper Rodlets." International Journal of Heat and Mass Transfer 79: 397-407. 
[7] Akihiro, T., Yasuhiro, T., Ryota, T., Shuji, O., and Kazuki, F. 2014. "Effects on the Initial Oxidation Mechanism of the Shot Peening Treatment for the Suppression of Surface Hot Shortness." Tetsu-to-Hagané 100 (3): 366-75.
[8] LI, X. G., and HE, J. W. 2006. Material Letters 60: 339.

[9] Kim, D. E., Yu, D. I., Jerng, D. W., Kim, M. H., and Ahn, H. S. 2015. "Review of Boiling Heat Transfer Enhancement on Micro/Nanostructured Surfaces." Experimental Thermal and Fluid Science 66: 173-96. 